\title{
miR-145-5p attenuates paclitaxel resistance and suppresses the progression in drug-resistant breast cancer cell lines
}

\author{
X. GUAN ${ }^{1, *}$, Y. GUAN ${ }^{2}$ \\ ${ }^{1}$ Department of Breast and Thyroid Surgery, Zaozhuang Municipal Hospital, Longtou Road No. 41, Shizhong District, China; ${ }^{2}$ Department of \\ Traditional Chinese Medicine, Taozhuang Town Central Hospital, Zaozhuang, China \\ *Correspondence: fhpnlj@163.com
}

Received June 22, 2019 / Accepted December 13, 2019

\begin{abstract}
miR-145-5p has been identified as a tumor suppressor involved in a wide variety of human cancers. Herein, we aimed to further explore the functional role and molecular mechanism of miR-145-5p on breast cancer (BC) progression and chemoresistance. The expressions of miR-145-5p and sex determining region Y-box2 (SOX-2) mRNA were assessed by qRT-PCR assay. SOX2 protein expression was measured using western blot. CCK-8 assays were used to determine the IC50 values of paclitaxel (PTX) and cell proliferation. Transwell assays were performed to detect cell migration and invasion. The target of miR-145-5p was verified by dual-luciferase reporter assays. Xenograft model was used to observe the role of miR-145-5p in vivo. The results showed that miR-145-5p was downregulated in BC tissues and cells and associated with PTX resistance of BC cells. Overexpression of miR-145-5p or SOX2 knockdown repressed the proliferation, migration, invasion, and attenuated PTX resistance in PTX-resistant BC cells. Mechanistically, miR-145-5p negatively regulated SOX2 expression by targeting SOX2. The inhibitory effects of miR-145-5p on the proliferation, migration, invasion, and PTX resistance were antagonized by SOX2 level restoration in PTX-resistant BC cells. Additionally, miR-145-5p repressed tumor growth in vivo. In conclusion, our study suggested that miR-145-5p reduced PTX resistance and repressed the progression at least partly by targeting SOX2 in PTX-resistant BC cells, highlighting miR-145-5p as a promising biomarker for BC treatment.
\end{abstract}

Key words: miR-145-5p, sex determining region Y-box2 (SOX-2), paclitaxel (PTX) resistance, drug-resistant BC cells

Breast cancer $(\mathrm{BC})$ is the most diagnosed malignancy and the leading cause of cancer-related death among women, with an estimated 2.1 million newly diagnosed cases in 2018 worldwide [1]. In recent decades, an increasing trend of $\mathrm{BC}$ morbidity has been observed in China, accounting for $15 \%$ of all new cancers in women [2]. Although the developments of systemic therapeutic strategies have improved the treatment effect of $\mathrm{BC}$, metastasis, and chemoresistance still are the main obstacles in BC management $[3,4]$. Therefore, it is of interest to identify novel biomarkers for BC treatment.

MicroRNAs (miRNAs), a class of small non-coding transcripts of 19-25 nucleotides, function as a translational repressors by binding to complementary sites in the 3'-untranslated regions (3'-UTR) of their target mRNAs [5]. Accumulating evidence has suggested that miRNAs control a broad array of pathological behaviors of cancer cells, such as cell proliferation, invasion, metastasis, and chemoresistance [6-8]. miR-145-5p has been identified as a tumor suppressor in a wide variety of human cancers, including lung squamous cell carcinoma [9], bladder cancer [10], and prostate cancer
[11]. miR-145-5p overexpression was reported to be able to sensitize cancer cells to drugs $[12,13]$. Moreover, low miR-145-5p expression was found in BC tissues and cells [14, 15]. miR-145-5p inhibited cell migration in the presence of Ago2, and its overexpression repressed BC development [16, 17]. Herein, we aimed to further explore the functional role and molecular mechanism of miR-145-5p on BC progression and chemoresistance.

In this study, our data validated that miR-145-5p was downregulated in BC and associated with PTX resistance of BC cells. Furthermore, miR-145-5p repressed the proliferation, migration, invasion, and attenuated paclitaxel (PTX) resistance at least partly by the regulation of sex determining region Y-box2 (SOX-2) in PTX-resistant BC cells.

\section{Materials and methods}

Clinical samples. A total of 32 pairs of BC tissues and corresponding noncancerous tissues were obtained from BC patients who had undergone surgical resection between 2015 
to 2017 at the Department of Breast and Thyroid Surgery, Zaozhuang Municipal Hospital. All tissues were collected by a core biopsy prior to treatment and immediately stored at $-80^{\circ} \mathrm{C}$ until RNA extraction. The clinicopathologic features of these patients are presented in Table 1. Written informed consent was obtained from all patients before surgery, and the permission for the study was obtained from the Institutional Review Board of Department of Breast and Thyroid Surgery, Zaozhuang Municipal Hospital.

Cell culture and treatment. Human normal breast epithelial cell line MCF-10A and two BC cells (MCF-7 and MDA-MB-231) were purchased from ATCC (Manassas, VA, USA). MCF-10A cells were maintained in DMEM/F-12 medium (Gibco, Carlsbad, CA, USA), and MCF-7 and MDA-MB-231 cells were maintained in DMEM medium (Gibco), supplemented with 10\% fetal bovine serum (FBS, Gibco), $1 \%$ penicillin/streptomycin (Gibco) at $37^{\circ} \mathrm{C}$ in a humidified atmosphere with $5 \% \mathrm{CO}_{2}$.

To establish PTX-resistant BC cells (MCF-7/PTX and MDA-MB-231/PTX), MCF-7 and MDA-MB-231 cells were treated with a stepwise increasing concentration of PTX (Sigma-Aldrich, Rehovot, Israel) for more than 6 months as described previously [18]. To maintain their PTX-resistant phenotype, an additional $0.5 \mu \mathrm{M}$ of PTX was added into the growth medium.

Cell transfection. Mature miR-145-5p mimics and negative control miRNA mimics (miR-NC mimics), miR-145-5p inhibitor (anti-miR-145-5p) and inhibitor control (anti-miR-NC), siRNA targeting SOX2 (si-SOX2) and negative control siRNA (si-NC), SOX2 overexpression plasmid (pcDNA-SOX2) and negative plasmid (pcDNANC) were purchased from GenePharma (Shanghai, China). Cells were transfected with the indicated oligonucleotide (at a final concentration of $50 \mathrm{nM}$ ) or $40 \mathrm{ng}$ of plasmid using X-treme Gene HP DNA Transfection Kit (Roche, Penzberg, Germany) according to the instruction of the manufacturers.

RNA extraction, reverse transcription, and quantitative real-time PCR (qRT-PCR). Total RNA was extracted from tissues and cells using a miRVana miRNA extraction

Table 1. The clinicopathological parameters of BC patients.

\begin{tabular}{lc}
\hline Variable & Patients, $\mathbf{n}$ \\
\hline Age, years & 32 \\
$\quad<45$ & 17 \\
$\quad \geq 45$ & 15 \\
Tumor size & \\
$\quad<2$ & 20 \\
$\geq 2$ & 12 \\
TNM stage & \\
$\quad<$ III stage & 15 \\
$\geq$ III stage & 17 \\
Lymph node status & \\
Yes & 21 \\
No & 11 \\
\hline
\end{tabular}

kit (Ambion, Austin, TX, USA) following the manufacturers' guidance, and then quantified by a NanoDrop spectrophotometer (NanoDrop Technologies, Wilmington, DE, USA). For SOX2 mRNA detection, RNA was reverse-transcribed into cDNA with the High-Capacity cDNA Reverse Transcription kit (Applied Biosystems, Foster City, CA, USA), and qRT-PCR was performed using a SYBR Green Master Mix (Applied Biosystems) on a PRISM 7700 Sequence Detector System (Applied Biosystems). For miR-145-5p detection, reverse transcription was performed using TaqMan Reverse Transcription kit (Applied Biosystems), and qRT-PCR was performed using TaqMan MicroRNA Assay kit (Applied Biosystems). For normalization of the target, GAPDH or U6 was used as an internal control. Gene expression data were calculated by the $2^{-\Delta \Delta C t}$ method.

IC50 detection and cell proliferation assay. The IC50 values and cell proliferation were assessed using a Cell Counting kit-8 (CCK-8, Dojindo Laboratories, Kumamoto, Japan) referring to the protocol of manufacturers. Briefly, cells were seeded into 96-well plates. At the indicated time, $10 \mu \mathrm{l}$ of CCK- 8 solution was added into each well and incubated for $2 \mathrm{~h}$ at $37^{\circ} \mathrm{C}$. The absorbance at $450 \mathrm{~nm}$ was determined by a microplate reader (Spectrostar Omega, BMG Labtech, Offenburg, Germany).

For IC50 values measurement, cells were seeded into 96-well plates, and increasing concentration of PTX $(0,0.5$, $1.0,2.0,4.0,8.0,16.0 \mu \mathrm{M})$ was added into each well. After $24 \mathrm{~h}$ incubation, CCK-8 solution was added, and the absorbance was detected.

Transwell assay of cell migration and invasion. For migration assay, cells were seeded into the upper chamber of a 24-Transwell plate with $8 \mu \mathrm{m}$ pores membrane inserts without Matrigel (Corning Incorporated, Tewksbury, MA, USA) precoating. For invasion assay, cells were added into the upper chamber of a 24-Transwell plate with a Matrigelprecoated membrane (Corning Incorporated). In both assays, $500 \mu \mathrm{l}$ of DMEM medium supplemented with $10 \%$ FBS was added into the lower chamber as a chemotactic agent. After $24 \mathrm{~h}$ incubation, migrated or invaded cells were fixed in 3.7\% formaldehyde and stained with $0.1 \%$ crystal violet. Images were photographed under a Nikon microscope (Nikon, Shinagawa, Tokyo, Japan) and the number of migrated or invaded cells was counted in five random fields.

Dual-luciferase reporter assay. Bioinformatics analysis for the targets of miR-145-5p was performed using miRTarBase software (http://mirtarbase.mbc.nctu.edu.tw/php/ search.php). SOX2 3'-UTR-luciferase reporters harboring the target sequence for miR-145-5p (SOX2 3'-UTR-WT) and site-directed mutation of the target sequence (SOX2 3'-UTRMUT) were obtained from GenePharma. Cells were co-transfected with SOX2 3'-UTR-WT or SOX2 3'-UTR-MUT and miR-NC mimics, miR-145-5p mimics, anti-miR-NC, or anti-miR-145-5p. After $24 \mathrm{~h}$ transfection, luciferase activities were measured by using the Dual-Luciferase Reporter Assay System (Dual-Light System, Applied Biosystems). 
Western blot for SOX2. Cells were lysed in lysis buffer comprised of $50 \mathrm{mM}$ Tris- $\mathrm{HCl}, \mathrm{pH} 7.4,150 \mathrm{mM} \mathrm{NaCl}, 1 \%$ Triton X-100, 0.1\% SDS, 1 mM EDTA, 0.5\% sodium deoxycholate and $1 \%$ protease inhibitor cocktails (Sigma-Aldrich). The concentration of protein samples was determined by a BCA Protein Assay kit (Sigma-Aldrich). Equal amounts of protein samples were separated by gel electrophoresis on $10 \%$ gels, transferred onto Hybond-P PVDF membranes (Amersham Biosciences, Buckinghamshire, UK) and then probed with primary antibodies including anti-SOX2 (Cell Signaling Technology, Danvers, MA, USA; dilution 1:1000) and anti- $\beta$-actin (Cell Signaling Technology; dilution 1:1000). Following the incubation with HRP-conjugated secondary antibodies (Cell Signaling Technology; dilution 1:5000), the protein bands were visualized with a chemiluminescence system (Amersham Biosciences) and quantified using ImageJ software (National Institutes of Health, Bethesda, MD, USA).

In vivo assay. All animal experimental processes were conducted in accordance with a protocol approved by the Institution Animal Care and Use Committee at the Department of Breast and Thyroid Surgery, Zaozhuang Municipal Hospital. MCF-7/PTX cells $\left(5 \times 10^{6}\right)$ were subcutaneously injected into BALB/c nude mice ( 4 weeks, Laboratory Animal Center of Henan Province, Zhengzhou, Henan, China). After implantation for 2 days, intratumor injection of miR-145-5p mimics or miR-NC mimics was performed every 3 days $(\mathrm{n}=10) .28$ days after implantation, all mice were euthanized, and xenograft tissues were excised for weight, qRT-PCR and western blot.

Statistical analysis. Data were analyzed using SPSS 18.0 software (SPSS Inc. Chicago, IL, USA) with the MannWhitney U-test, a student's t-test, and ANOVA. All data were presented as mean $\pm \mathrm{SD}$, and statistical significance was defined as $\mathrm{p}<0.05$.

\section{Results}

miR-145-5p was downregulated in BC and associated with PTX resistance of BC cells. Firstly, miR-145-5p expression was determined in $\mathrm{BC}$ tissues and corresponding noncancerous tissues by qRT-PCR assay. These data revealed that miR-145-5p was significantly downregulated in $\mathrm{BC}$ tissues compared with normal tissues (Figure 1A). To investigate the role of miR-145-5p on PTX resistance of BC, two PTX-resistant BC cell lines (MCF-7/PTX and MDA-MB-231/PTX) were established. Then, the expression of miR-145-5p was assessed in MCF-7/PTX, MDA-MB231/PTX, and their parental cells. These results showed that compared with MCF-10A cells, miR-145-5p expression levels were significantly decreased in MCF-7 and
A

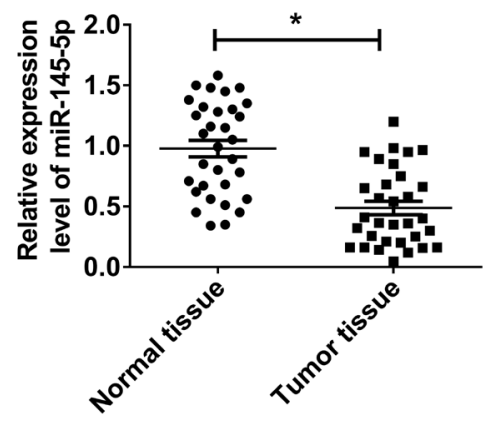

C

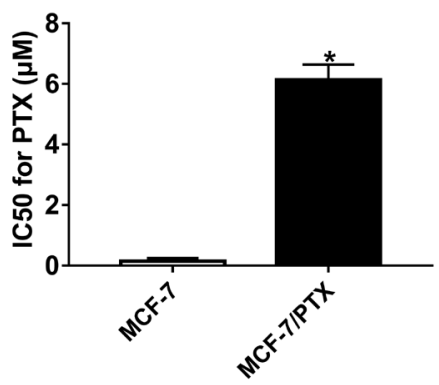

B

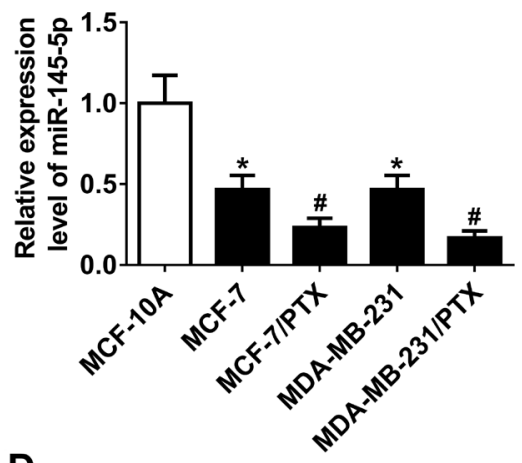

D

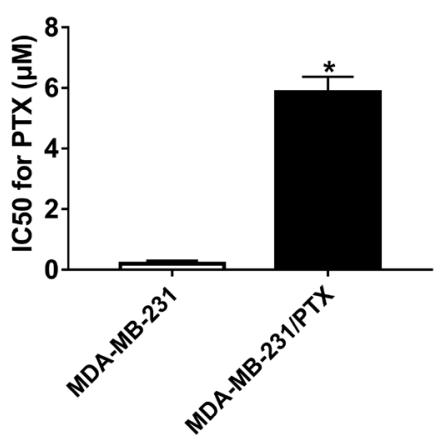

Figure 1. miR-145-5p was downregulated in BC tissues and associated with PTX resistance of BC cells. The expression levels of miR-145-5p were determined by $\mathrm{qRT}$-PCR in 32 pairs of BC tissues and corresponding noncancerous tissues (A), MCF-10A, MCF-7, MDA-MB-231, MCF-7/PTX, and MDAMB-231/PTX cells (B). The IC50 values of PTX were detected by CCK-8 assay in MCF7 and MCF7/PTX cells (C), MDA-MB-231, and MDA-MB-231/ PTX cells (D). ${ }^{\star} \mathrm{p}<0.05$ or ${ }^{*} \mathrm{p}<0.05$ 
MDA-MB-231 cells (Figure 1B). Moreover, PTX treatment resulted in a more distinct reduction of miR-145-5p expression (Figure 1B). Meanwhile, we detected the IC50 values of PTX in MCF-7/PTX, MDA-MB-231/PTX, and their parental cells. As expected, the IC50 values of PTX in MCF-7/PTX and MDA-MB-231/PTX cells were higher than those in their parental cells (Figures 1C, 1D).

miR-145-5p overexpression repressed the proliferation, migration, invasion, and attenuated PTX resistance in PTX-resistant BC cells. To explore the role of miR-145-5p on BC, gain-of-function experiments were performed by transfection of miR-145-5p mimics. In contrast to the negative control, transient transfection of miR-145-5p mimics resulted in a significant upregulation of miR-145-5p expression in MCF-7/PTX and MDA-MB-231/PTX cells (Figure 2A). Subsequently, CCK-8 assays revealed that in comparison to the negative control, miR-145-5p overexpression markedly reduced the IC50 values of PTX and repressed the proliferation in MCF-7/PTX and MDA-MB-231/PTX cells (Figures 2B-2D). Moreover, transwell assays showed that miR-145-5p overexpression significantly repressed cell migration and invasion compared to the negative control (Figures 2E, 2F).

SOX2 was a direct target of miR-145-5p. To further understand the effect of miR-145-5p on BC, we carried out a detailed analysis of its molecular targets by miRTar-
Base software (http://mirtarbase.mbc.nctu.edu.tw/php/ search.php). The predicted data revealed that the 3'-UTR of SOX2 contained a putative target sequence for miR-145-5p (Figure 3A). To confirm this, SOX2 3'-UTR-luciferase reporter (SOX2 3'-UTR-WT) harboring the target sequence for miR-145-5p and site-directed mutation of target sequence (SOX2 3'-UTR-MUT) were used in luciferase assays. In comparison to respective control, the luciferase activity of SOX2 3'-UTR-WT was significantly decreased by transfection of miR-145-5p mimics, while it was highly increased in the presence of anti-miR-145-5p (Figures 3B-3E). However, the site-directed mutation of the target sequence abolished the effect of miR-145-5p on luciferase activity under the same conditions (Figures 3B-3E).

Then, we determined whether, and if so, how miR-145-5p modulated SOX2 expression in $\mathrm{BC}$ cells. In contrast to their counterparts, SOX2 protein expression was significantly repressed by miR-145-5p overexpression, while it was strongly enhanced following the miR-145-5p knockdown, thus indicating that miR-145-5p was able to negatively modulate SOX2 expression in BC cells (Figure 3F). Additionally, qRT-PCR results revealed that compared with the negative group, little change was observed in miR-145-5p expression in the case of si-SOX2 transfection in the two BC cells (Figure 3G). Subsequently, SOX2 protein expression was assessed in MCF-7/PTX, MDA-MB-231/PTX, and their
A

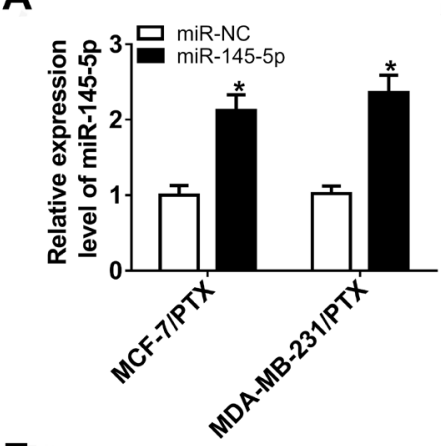

$\mathbf{E}$

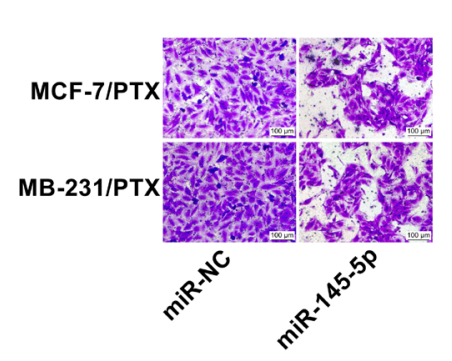

B

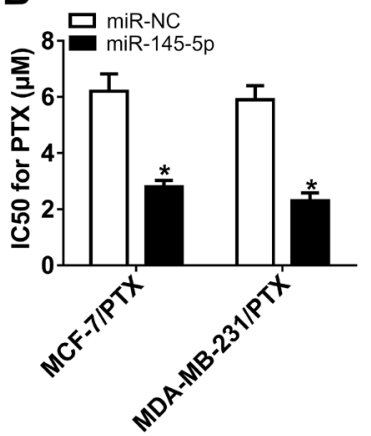

$\mathbf{F}$

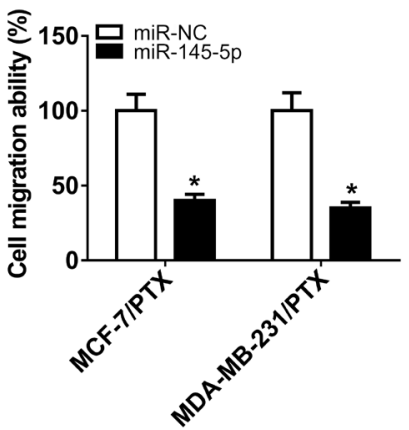

C
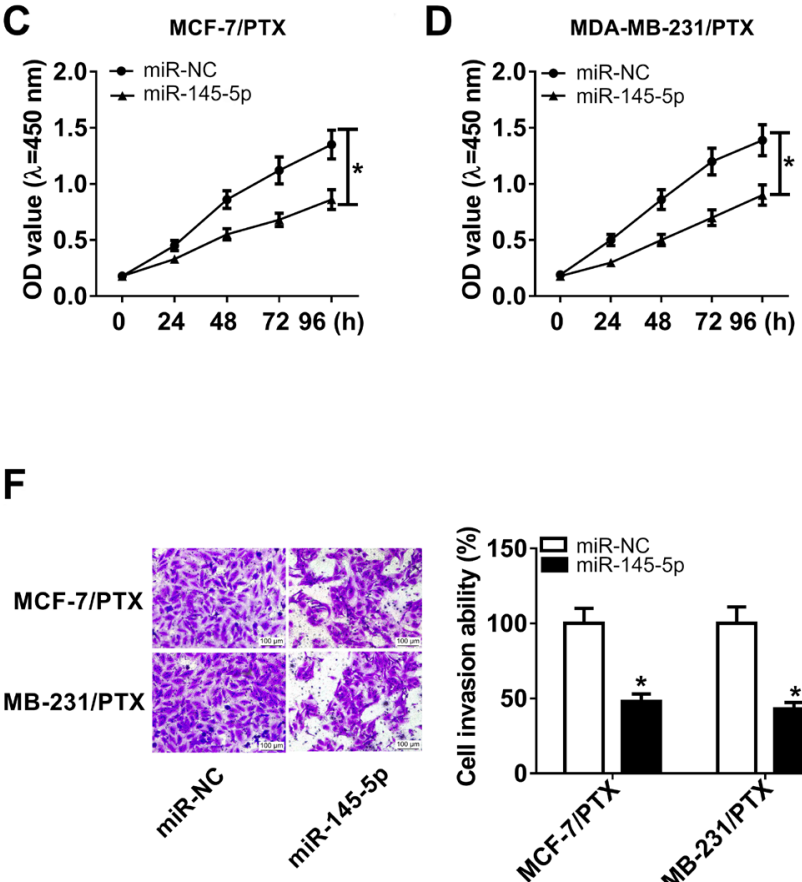

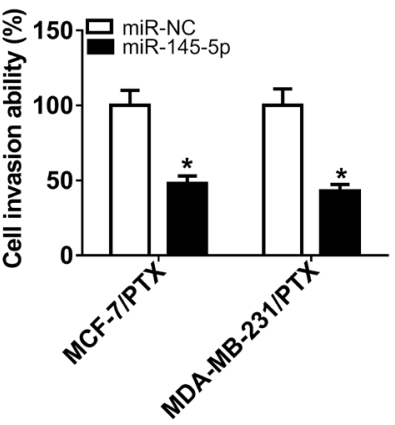

Figure 2. miR-145-5p repressed the proliferation, migration, invasion, and PTX resistance in BC cells. MCF-7/PTX and MDA-MB-231/PTX cells were transfected with miR-145-5p mimics or miR-NC mimics, followed by the measurement of miR-145-5p expression by qRT-PCR assay (A), the IC50 values of PTX by CCK-8 assay (B), cell proliferation ability by CCK-8 assay (C) and (D), cell migration ability (E), and invasion capacity (F) by transwell assay. ${ }^{*} \mathrm{p}<0.05$ 
A

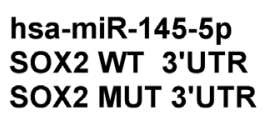

B

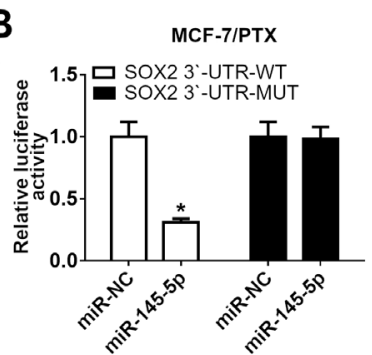

$\mathbf{F}$

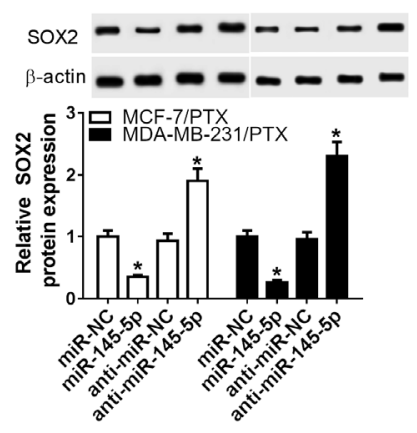

\section{3' UCCCUAAGgACCCUUUUGACCUG 5' $5 '$...GGGCCGGACAGCGAACUGGAG... 3' 5 ' ...GGGCCGgaCAGCGUUGACCUG... 3'}

C

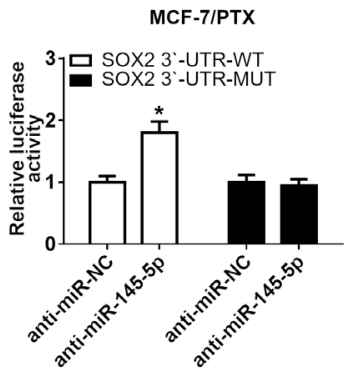

D

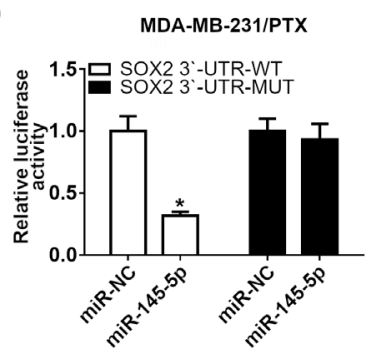

E

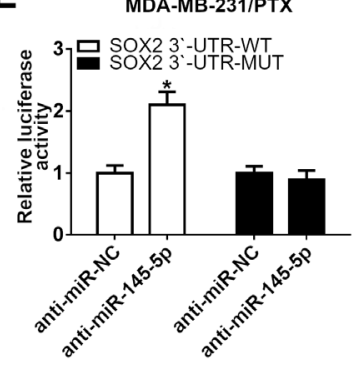

G

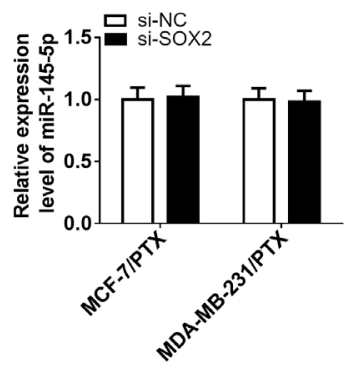

H

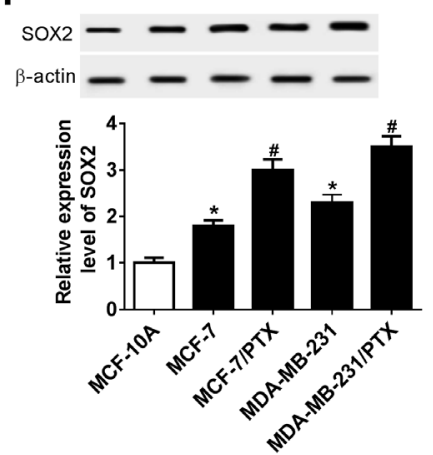

Figure 3. SOX2 was a direct target of miR-145-5p in BC cells. (A) Predicted target sequence between miR-145-5p and SOX2 3'-UTR, and site-directed mutation of the target sequence. MCF-7/PTX cells (B and C) and MDA-MB-231/PTX cells (D and E) were co-transfected with SOX2 3'-UTR-WT or SOX2 3'-UTR-MUT and miR-NC mimics, miR-145-5p mimics, anti-miR-NC, or anti-miR-145-5p, followed by the detection of the luciferase activities. (F) MCF-7/PTX and MDA-MB-231/PTX cells were transfected with miR-NC mimics, miR-145-5p mimics, anti-miR-NC, or anti-miR-145-5p, followed by the measurement of SOX2 protein expression by western blot. (G) MCF-7/PTX and MDA-MB-231/PTX cells were transfected with si-NC or si-SOX2, followed by the detection of miR-145-5p expression by qRT-PCR. (H) The expression of SOX2 protein was determined in MCF-10A, MCF-7, MDA-MB-231, MCF-7/PTX, and MDA-MB-231/PTX cells. ${ }^{*} \mathrm{p}<0.05$ or ${ }^{*} \mathrm{p}<0.05$

parental cells. Western blot results revealed that SOX2 levels were highly upregulated in MCF-7 and MDA-MB-231 cells compared with MCF-10A cells (Figure 3H). Moreover, PTX treatment led to a more obvious upregulation of the SOX2 level in BC cells (Figure 3H).

SOX2 knockdown inhibited the proliferation, migration, and invasion, and reduced PTX resistance in PTX-resistant BC cells. Further, we observed the role of SOX2 on BC by transfection of si-SOX2. In comparison to the negative control, the transient introduction of si-SOX2 led to a strong reduction of SOX mRNA and protein levels in MCF-7/PTX and MDA-MB-231/PTX cells (Figures 4A, 4B). CCK-8 assays showed that SOX2 knockdown substantially attenuated the IC50 values of PTX and highly inhibited the proliferation in MCF-7/PTX and MDA-MB-231/PTX cells (Figures 4C-4E). Transwell assays revealed that cell migration and invasion abilities were significantly repressed by SOX 2 knockdown compared with the negative control (Figures 4F, 4G).
SOX2 level restoration antagonized the inhibitory effects of miR-145-5p on the proliferation, migration, invasion, and PTX resistance in PTX-resistant BC cells. To provide further mechanistic insight into the link between miR-145-5p and SOX2 on BC, BC cells were co-transfected with miR-145-5p mimics and pcDNA-SOX2. Western blot data revealed that compared with the negative control, the co-transfection of pcDNA-SOX2 significantly abrogated the inhibitory effect of miR-145-5p on SOX2 expression (Figure 5A). CCK-8 assays revealed that the reduced effect of miR-145-5p on PTX resistance was also strikingly reversed by the SOX2 level restoration (Figure 5B). Further functional experiments demonstrated that miR-145-5p-mediated antiproliferation, anti-migration, and anti-invasion effects were markedly abrogated by the SOX2 level restoration in MCF-7/ PTX and MDA-MB-231/PTX cells (Figures 5C-5F).

miR-145-5p overexpression suppressed tumor growth in vivo. Next, to investigate the role of miR-145-5p on $\mathrm{BC}$ in vivo, mice xenograft models were prepared by subcutaneous 
injection of MCF-7/PTX cells. These results revealed that the introduction of miR-145-5p mimics highly suppressed tumor growth compared with the negative control (Figures 6A, 6B). Moreover, miR-145-5p expression was increased (Figure 6C), while SOX2 mRNA and protein levels were decreased (Figures 6D, 6E) in xenograft tissues derived from miR-1455p-administered MCF-7/PTX cells.

\section{Discussion}

Up to now, many miRNAs have been identified as oncogenes or tumor suppressors in BC. For example, miR-21 enhanced the proliferation and invasion of triple-negative
BC cells via targeting PTEN [19]. miR-181a promoted aggressive behavior of $\mathrm{BC}$ cells and chemotherapy resistance [20]. Conversely, miR-134 was downregulated in BC, and miR-134 overexpression inhibited the proliferation and enhanced apoptosis on BC cells [21]. Ectopic overexpression of miR-621 enhanced cell apoptosis and sensitized cell to PTX and carboplatin through inhibition of FBXO11 and enhancement of p53 activity in BC cells [22]. These miRNAs might serve as potential predictive biomarkers and therapeutic targets for $\mathrm{BC}$ treatment.

Downregulation of miR-145-5p has been found in a series of human cancers, and previous studies have highlighted its role as a tumor suppressor by modulating several types of
A

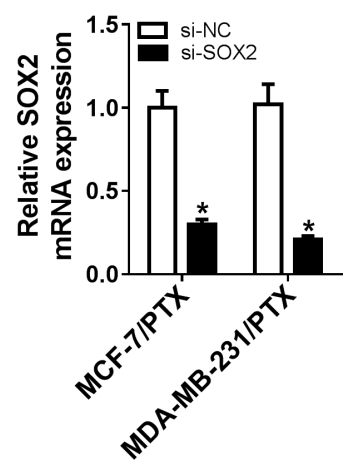

D

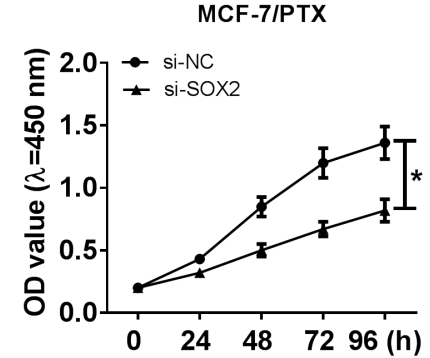

$\mathbf{F}$

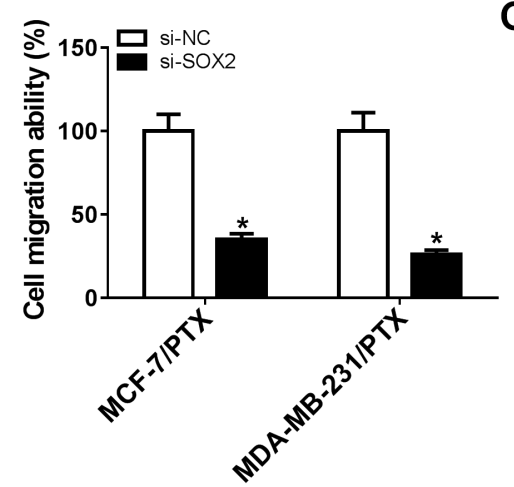

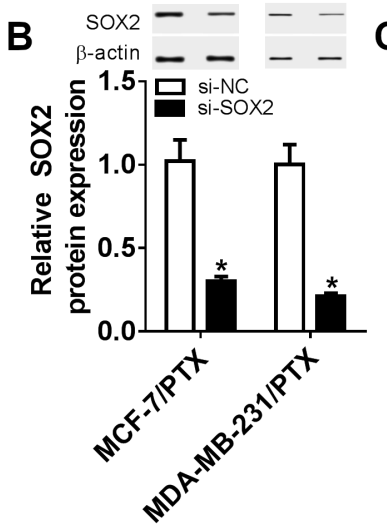

C

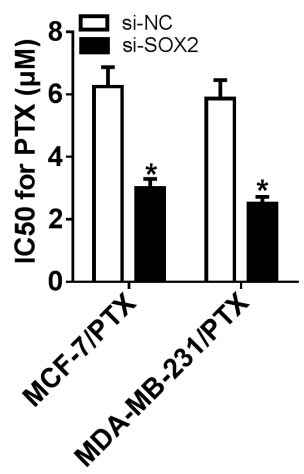

E

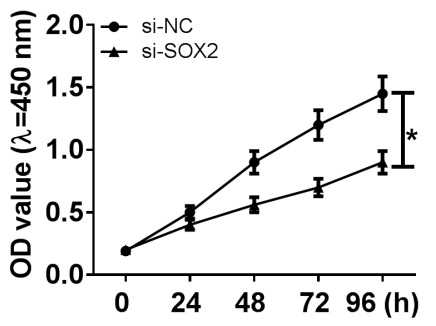

G

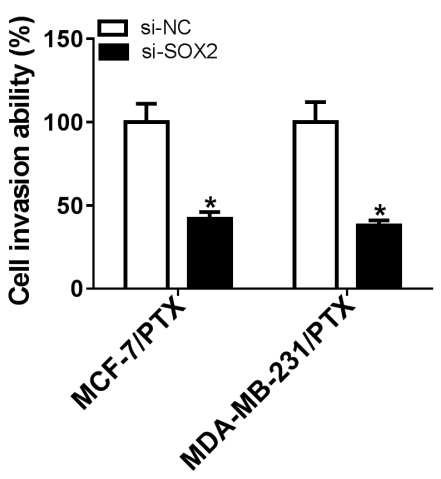

Figure 4. SOX2 knockdown inhibited the proliferation, migration, and invasion, and reduced PTX resistance of PTX-resistant BC cells. MCF-7/PTX and MDA-MB-231/PTX cells were transfected with si-NC or si-SOX2, followed by the determination of SOX2 mRNA expression by qRT-PCR assay (A), SOX2 protein level by western blot (B), the IC50 values of PTX (C) and cell proliferation (D and E) by CCK-8 assay, cell migration ability (F), and invasion capacity (G) by transwell assay. ${ }^{*} \mathbf{p}<0.05$ 

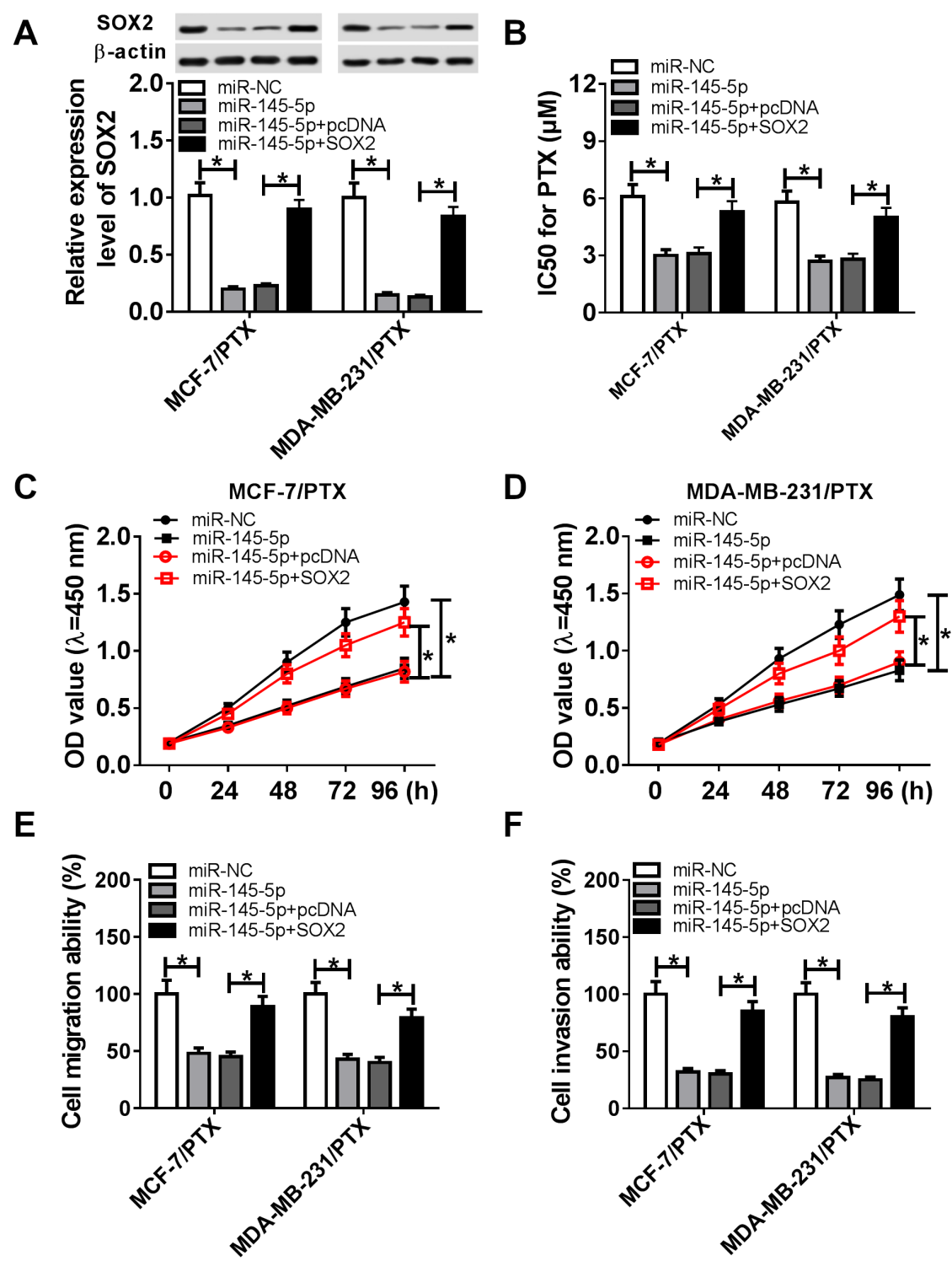

Figure 5. SOX2 level restoration antagonized the inhibitory effects of miR-145-5p on the proliferation, migration, invasion, and PTX resistance in PTX-resistant BC cells. MCF-7/PTX and MDA-MB-231/PTX cells were transfected with miR-NC mimics, miR-145-5p mimics, miR-145-5p mimics+pcDNA-NC or miR-145-5p mimics+pcDNA-SOX2, followed by the measurement of SOX2 expression by western blot (A), the IC50 values of PTX (B), and cell proliferation (C and D) by CCK-8 assay, cell migration (E), and invasion (F) abilities by transwell assay. ${ }^{*}$ p $<0.05$

oncogenes in cancer cells [23-25]. The loss of the miR-143/ miR-145 cluster promoted the proliferation and invasion of renal cell carcinoma cells via targeting hexokinase-2 [26]. The transfer of miR-145-5p from microvascular endothelial cells to cancer cells repressed angiogenesis through regulation of composed of connexin-43 in colon cancer cells [27]. Moreover, miR-145-5p enhanced the PTX resistance of ovarian cancer cells through targeting Sp1 and Cdk6 [28]. In the present study, our data supported that miR-145-5p was downregulated in $\mathrm{BC}$ tissues and cells, in accordance with past studies [16, 29]. Moreover, we firstly demonstrated that downregulation of miR-145-5p might be associated with PTX resistance of BC cells. Further, we verified that miR-145-5p overexpression repressed the proliferation, migration, invasion, and attenuated PTX resistance in PTX-resistant BC cells. These results strongly pointed to the role of miR-145-5p as a tumor suppressor in BC.

Then, miRTarBase software was performed to predict the molecular targets of miR-145-5p. Among these candidates, SOX2 was selected for further experiments, considering its crucial roles of oncogenesis in many varieties of human cancers, such as prostate cancer [30], gastric carcinoma [31], and melanoma [32]. Additionally, SOX2 was demonstrated to control tumor initiation and progression by modulating 
A

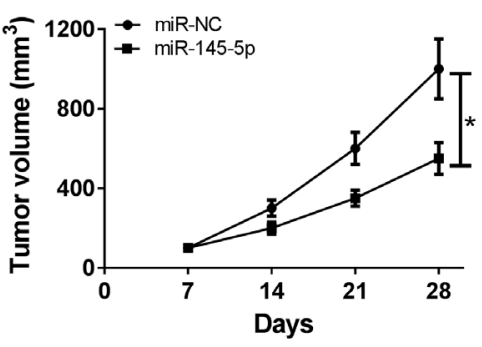

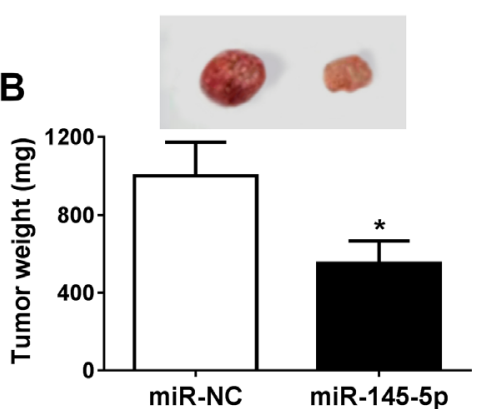

E

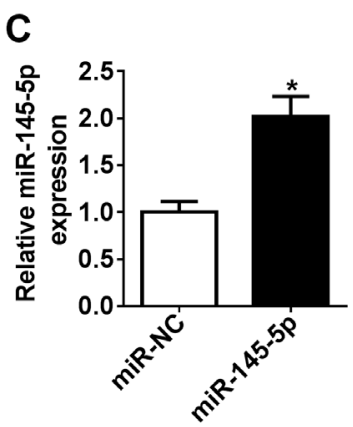

D
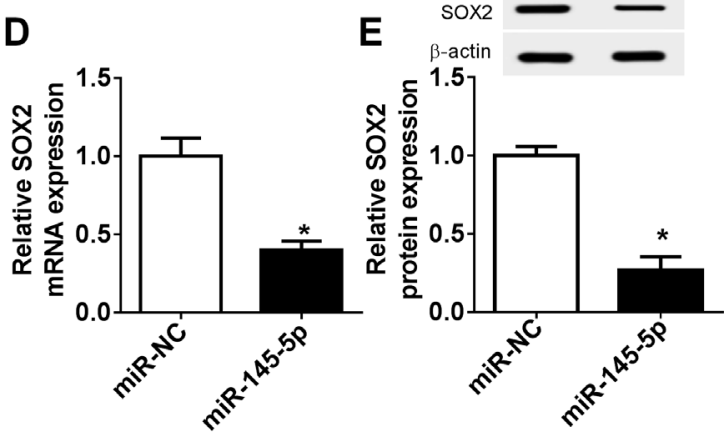

Figure 6. miR-145-5p suppressed tumor growth in vivo. Nude mice were subcutaneously injected with about $5 \times 10^{6} \mathrm{MCF}-7 / \mathrm{PTX}$ cells. After implantation for 2 days, miR-145-5p mimics or miR-NC mimics were administered by intratumoral injection every 3 days. 28 days after implantation, all mice were euthanized. (A) After implantation for 7 days, tumor volume was measured every 7 days. (B) Representative images and tumor tissues were weighed. qRT-PCR assay for miR-145-5p expression (C) and SOX2 mRNA level (D), and western blot for SOX2 protein level (E) in excised tumor tissues. ${ }^{*} \mathrm{p}<0.05$

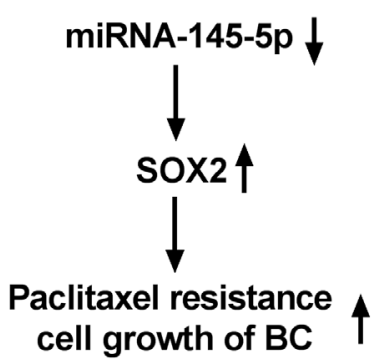

Figure 7. The mechanism schematic model by the miR-145-5p/SOX2 axis in BC. The expression of miR-145-5p was downregulated in BC. Then, the SOX2 level was upregulated. Upregulated SOX2 promoted BC cell resistance to PTX.

the functions of skin tumor initiating cells and cancer stem cells in skin squamous cell carcinoma [33]. High SOX2 expression was frequently observed in small-cell lung cancer tissues, and SOX2 depletion repressed cancer cell proliferation [34]. Subsequently, we confirmed that SOX2 was a direct target of miR-145-5p, and miR-145-5p negatively regulated SOX2 expression in BC cells. Similar findings indicated that upregulated miR-145-5p inhibited the expression of SOX2 in prostate cancer cells [11]. Moreover, SOX2 accelerated the proliferation and tumorigenesis via regulating CCND1 expression in BC cells [35]. SOX2 knockdown hampered mammosphere formation and retarded tumor formation in vivo models, providing the evidence for $\mathrm{SOX} 2$ reactivation as an early step in BC initiation [36]. In the present study, our results validated that SOX2 expression was strongly elevated in PTX-resistant BC cells. Furthermore, we manifested that SOX2 knockdown inhibited the proliferation, migration, and invasion and reduced PTX resistance in PTX-resistant $\mathrm{BC}$ cells. In a word, SOX2 functioned as an oncogene in BC, consistent with previous works $[35,36]$. miRNAs have been postulated to play crucial roles by modulating targets expression [5]. Therefore, we further explored whether miR-145-5p exerted its anti-tumor function by targeting SOX2 in BC. Our results demonstrated that SOX2 level restoration antagonized the inhibitory effects of miR-145-5p on the proliferation, migration, invasion, and PTX resistance in PTX-resistant $\mathrm{BC}$ cells.

Lastly, in vivo assays revealed that miR-145-5p inhibited tumor growth in mice xenograft models. Moreover, miR-145-5p overexpression repressed SOX2 expression, indicating that miR-145-5p repressed tumor growth through targeting SOX2 in vivo.

In conclusion, our study suggested that miR-145-5p reduced PTX resistance and repressed the progression at least partly by targeting SOX2 in PTX-resistant BC cells. Targeting miR-145-5p may be a promising biomarker for BC treatment. 


\section{References}

[1] BRAY F, FERLAY J, SOERJOMATARAM I, SIEGEL RL, TORRE LA et al. Global cancer statistics 2018: GLOBOCAN estimates of incidence and mortality worldwide for 36 cancers in 185 countries. CA Cancer J Clin 2018; 68: 394-424. https://doi.org/10.3322/caac.21492

[2] CHEN W, ZHENG R, BAADE PD, ZHANG S, ZENG H et al. Cancer statistics in China, 2015. CA Cancer J Clin 2016; 66: 115-132. https://doi.org/10.3322/caac.21338

[3] WANG D, KHOSLA A, GARGEYA R, IRSHAD H, BECK $\mathrm{AH}$. Deep learning for identifying metastatic breast cancer. arXiv:1606.057182016.

[4] VElagA R, SUGiMOTO M. Future Paradigm of Breast Cancer Resistance and Treatment, pp 155-178. In: J. Prosperi (Ed.). Resistance to Targeted Therapies in Breast Cancer. Springer International Publishing 2017, p 184. ISBN 978-3319-70142-4

[5] IWAKAWA HO, TOMARI Y. The Functions of MicroRNAs: mRNA Decay and Translational Repression. Trends Cell Biol 2015; 25: 651-665. https://doi.org/10.1016/j.tcb.2015.07.011

[6] HAYES J, PERUZZI PP, LAWLER S. MicroRNAs in cancer: biomarkers, functions and therapy. Trends Mol Med 2014; 20: 460-469. https://doi.org/10.1016/j.molmed.2014.06.005

[7] BLOWER PE, CHUNG JH, VERDUCCI JS, LIN S, PARK JK et al. MicroRNAs modulate the chemosensitivity of tumor cells. Mol Cancer Ther 2008; 7: 1-9. https://doi. org/10.1158/1535-7163.MCT-07-0573

[8] GILAM A, CONDE J, WEISSGLAS-VOLKOV D, OLIVA N, FRIEDMAN E et al. Local microRNA delivery targets Palladin and prevents metastatic breast cancer. Nat Commun 2016; 7: 12868. https://doi.org/10.1038/ncomms12868

[9] MATAKI H, SEKI N, MIZUNO K, NOHATA N, KAMIKAWAJI $\mathrm{K}$ et al. Dual-strand tumor-suppressor microRNA-145 (miR-145-5p and miR-145-3p) coordinately targeted MTDH in lung squamous cell carcinoma. Oncotarget 2016; 7: 72084-72098. https://doi.org/10.18632/oncotarget. 12290

[10] MATSUSHITA R, YOSHINO H, ENOKIDA H, GOTO Y, MIYAMOTO K et al. Regulation of UHRF1 by dual-strand tumor-suppressor microRNA-145 (miR-145-5p and miR145-3p): Inhibition of bladder cancer cell aggressiveness. Oncotarget 2016; 7: 28460-28487. https://doi.org/10.18632/ oncotarget. 8668

[11] OZEN M, KARATAS OF, GULLUOGLU S, BAYRAK OF, SEVLI S et al. Overexpression of miR-145-5p inhibits proliferation of prostate cancer cells and reduces SOX2 expression. Cancer Invest 2015; 33: 251-258. https://doi.org/10.3109/07 357907.2015.1025407

[12] XUE D, LU H, XU HY, ZHOU CX, HE XZ. Long noncoding RNA MALAT 1 enhances the docetaxel resistance of prostate cancer cells via miR-145-5p-mediated regulation of AKAP 12. J Cell Mol Med 2018; 22: 3223-3237. https://doi. org/10.1111/jcmm.13604

[13] JIAN M, DU Q, ZHU D, MAO Z, WANG X et al. Tumor suppressor miR-145-5p sensitizes prolactinoma to bromocriptine by downregulating TPT1. J Endocrinol Invest 2018. https://doi.org/10.1007/s40618-018-0963-4
[14] JIANG R, ZHAO C, GAO B, XU J, SONG W et al. Mixomics analysis of breast cancer: Long non-coding RNA linc01561 acts as ceRNA involved in the progression of breast cancer. Int J Biochem Cell Biol 2018; 102: 1-9. https://doi. org/10.1016/j.biocel.2018.06.003

[15] WANG S, XUE X, WANG R, LI X, LI Q et al. CircZNF609 promotes breast cancer cell growth, migration, and invasion by elevating $\mathrm{p} 70 \mathrm{~S} 6 \mathrm{~K} 1$ via sponging miR-145-5p. Cancer Manag Res 2018; 10: 3881-3890. https://doi.org/10.2147/ CMAR.S174778

[16] BELLISSIMO T, TITO C, GANCI F, SACCONI A, MASCIARELLI $S$ et al. Argonaute 2 drives miR-145-5p-dependent gene expression program in breast cancer cells. Cell Death Dis 2019; 10: 17. https://doi.org/10.1038/s41419-018-1267-5

[17] YE P, SHI Y, AN N, ZHOU Q, GUO J et al. miR-145 overexpression triggers alteration of the whole transcriptome and inhibits breast cancer development. Biomed Pharmacother 2018; 100: 72-82. https://doi.org/10.1016/j.biopha.2018.01.167

[18] SPROUSE AA, HERBERT BS. Resveratrol augments paclitaxel treatment in MDA-MB-231 and paclitaxel-resistant MDA-MB-231 breast cancer cells. Anticancer Res 2014; 34: 5363-5374.

[19] FANG H, XIE J, ZHANG M, ZHAO Z, WAN Y et al. miRNA-21 promotes proliferation and invasion of triple-negative breast cancer cells through targeting PTEN. Am J Transl Res 2017; 9: 953-961.

[20] NIU J, XUE A, CHI Y, XUE J, WANG W et al. Induction of miRNA-181a by genotoxic treatments promotes chemotherapeutic resistance and metastasis in breast cancer. Oncogene 2016; 35: 1302-1313. https://doi.org/10.1038/onc.2015.189

[21] O'BRIEN K, LOWRY MC, CORCORAN C, MARTINEZ VG, DALY M et al. miR-134 in extracellular vesicles reduces triple-negative breast cancer aggression and increases drug sensitivity. Oncotarget 2015; 6: 32774-32789. https://doi. org/10.18632/oncotarget.5192

[22] XUE J, CHI Y, CHEN Y, HUANG S, YE X et al. MiRNA-621 sensitizes breast cancer to chemotherapy by suppressing FBXO11 and enhancing p53 activity. Oncogene 2016; 35: 448-458. https://doi.org/10.1038/onc.2015.96

[23] LETELIER P, GARCIA P, LEAL P, ALVAREZ H, ILI C et al. miR-1 and miR-145 act as tumor suppressor microRNAs in gallbladder cancer. Int J Clin Exp Pathol 2014; 7: 1849-1867.

[24] XU W, CHANG J, DU X, HOU J. Long non-coding RNA PCAT-1 contributes to tumorigenesis by regulating FSCN1 via $\mathrm{miR}-145-5 \mathrm{p}$ in prostate cancer. Biomed Pharmacother 2017; 95: 1112-1118. https://doi.org/10.1016/j.biopha.2017.09.019

[25] MEI LL, WANG WJ, QIU YT, XIE XF, BAI J et al. miR-145$5 p$ Suppresses Tumor Cell Migration, Invasion and Epithelial to Mesenchymal Transition by Regulating the Sp1/NFkappaB Signaling Pathway in Esophageal Squamous Cell Carcinoma. Int J Mol Sci 2017; 18. https://doi.org/10.3390/ ijms 18091833

[26] YOSHINO $\mathrm{H}$, ENOKIDA $\mathrm{H}$, ITESAKO T, KOJIMA S, KINOSHITA T et al. Tumor-suppressive microRNA-143/145 cluster targets hexokinase-2 in renal cell carcinoma. Cancer Sci 2013; 104: 1567-1574. https://doi.org/10.1111/cas.12280 
[27] THURINGER D, JEGO G, BERTHENET K, HAMMANN A, SOLARY E et al. Gap junction-mediated transfer of miR145-5p from microvascular endothelial cells to colon cancer cells inhibits angiogenesis. Oncotarget 2016; 7: 28160-28168. https://doi.org/10.18632/oncotarget.8583

[28] ZHU X, LI Y, XIE C, YIN X, LIU Y et al. miR-145 sensitizes ovarian cancer cells to paclitaxel by targeting Sp1 and Cdk6. Int J Cancer 2014; 135: 1286-1296. https://doi.org/10.1002/ ijc. 28774

[29] TANG W, ZHANG X, TAN W, GAO J, PAN L, et al. miR145-5p Suppresses Breast Cancer Progression by Inhibiting SOX2. J Surg Res 2019; 236: 278-287. https://doi. org/10.1016/j.jss.2018.11.030

[30] JIA X, LI X, XU Y, ZHANG S, MOU W et al. SOX2 promotes tumorigenesis and increases the anti-apoptotic property of human prostate cancer cell. J Mol Cell Biol 2011; 3: 230-238. https://doi.org/10.1093/jmcb/mjr002

[31] ZHANG X, YU H, YANG Y, ZHU R, BAI J et al. SOX2 in gastric carcinoma, but not Hath1, is related to patients' clinicopathological features and prognosis. J Gastrointest Surg 2010; 14: 1220-1226. https://doi.org/10.1007/s11605-010$1246-3$
[32] GIROUARD SD, LAGA AC, MIHM MC, SCOLYER RA, THOMPSON JF et al. SOX2 contributes to melanoma cell invasion. Lab Invest 2012; 92: 362-370. https://doi. org/10.1038/labinvest.2011.188

[33] BOUMAHDI S, DRIESSENS G, LAPOUGE G, RORIVE S, NASSAR D et al. SOX2 controls tumour initiation and cancer stem-cell functions in squamous-cell carcinoma. Nature 2014; 511: 246-250. https://doi.org/10.1038/nature13305

[34] RUDIN CM, DURINCK S, STAWISKI EW, POIRIER JT, MODRUSAN $\mathrm{Z}$ et al. Comprehensive genomic analysis identifies SOX2 as a frequently amplified gene in small-cell lung cancer. Nat Genet 2012; 44: 1111-1116. https://doi. org/10.1038/ng.2405

[35] CHEN Y, SHI L, ZHANG L, LI R, LIANG J et al. The molecular mechanism governing the oncogenic potential of SOX2 in breast cancer. J Biol Chem 2008; 283: 17969-17978. https://doi.org/10.1074/jbc.M802917200

[36] LEIS O, EGUIARA A, LOPEZ-ARRIBILLAGA E, ALBERDI MJ, HERNANDEZ-GARCIA $S$ et al. Sox2 expression in breast tumours and activation in breast cancer stem cells. Oncogene 2012; 31: 1354-1365. https://doi.org/10.1038/ onc. 2011.338 